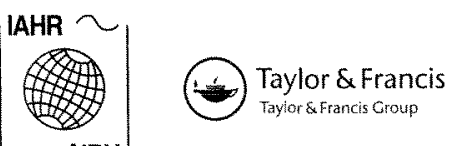

Journal of Hydraulic Research Vol. 49, No. 3 (2011), pp. 352-358

doi: $10.1080 / 00221686.2011 .574387$

(C) 2011 International Association for Hydro-Environment Engineering and Research

Research paper

\title{
Experimental study of velocity fields in rectangular shallow reservoirs
}

\author{
ERICA CAMNASIO (IAHR Young Member), PhD Student, Department of Hydraulic, Environmental, \\ Infrastructures and Surveying Engineering (D.I.I.A.R.), Politecnico di Milano (POLIMI), Piazza Leonardo da Vinci \\ 32, 20133 Milano, Italy. \\ Email: erica.camnasio@polimi.it (author for correspondence)
}

ENRICO ORSI (IAHR Member), Professor, Department of Hydraulic, Environmental, Infrastructures and Surveying Engineering (D.I.I.A.R.), Politecnico di Milano (POLIMI), Piazza Leonardo da Vinci 32, 20133 Milano, Italy.

Email: enrico.orsi@polimi.it

ANTON J. SCHLEISS (IAHR Member), Professor, Laboratory of Hydraulic Constructions (LCH), Ecole

Polytechnique Fédérale de Lausanne (EPFL), Station 18, 1015 Lausanne, Switzerland.

Email: anton.schleiss@epfl.ch

\begin{abstract}
Velocity fields in rectangular shallow reservoirs with different length-to-width and expansion ratios were investigated in an experimental study, to evaluate the effect of geometry on the flow field. A wide range of combinations of these two non-dimensional geometric parameters were tested at constant hydraulic conditions. Ultrasound velocity profilers were used to measure the horizontal velocity components across the entire reservoir surface, allowing for the visualization of streamlines and of the instantaneous and average velocities. Five different types of flow patterns were identified, depending on the values of the length-to-width ratio and expansion ratio of the reservoir. Asymmetrical flow patterns were found to develop for certain combinations of these geometric parameters despite the perfect reservoir symmetry. A critical comparison of these new experimental results with those of other works is provided.
\end{abstract}

Keywords: Flow pattern, geometrical effect, instability, large scale turbulent structure, shallow reservoir, velocity measurement

\section{Introduction}

The effects of the length-to-width ratio and of the expansion ratio, defined as the ratio between reservoir and inlet channel widths, on the flow pattern developing in a reservoir are not yet fully understood. Large-scale turbulent structures are strongly influenced by geometry (Versteeg and Malalasekera 1995) generating an asymmetric flow field despite a perfect reservoir symmetry for certain geometric and hydraulic conditions (Kantoush 2008).

The correct identification of the flow pattern, and a quantification of the velocities characterizing the main jet, the eddies and the recirculation zones, is important for engineering applications. Typical problems include sedimentation in shallow reservoirs (Kantoush and Schleiss 2009), Combined Sewer Overflow storage chambers (Stovin and Saul 1994) or settling tanks (Frey et al. 1993). As velocity governs the mechanisms of transport, sedimentation and re-suspension of the inflow, its accurate assessment would allow to predict the preferential sedimentation zones, leading to improved reservoir management. Precise flow-field characterization is also essential for appropriate design and management of aquaculture tanks (Oca et al. 2004), since the presence of dead zones with low water exchange would be detrimental to fish habitats. Furthermore, the flow pattern affects the actual detention time, and consequently the efficiency of shallow ponds, wetlands (Persson 2000) and dredged material containment areas (Thackston et al. 1987). Persson and Wittgren (2003) conducted numerical investigations of detention systems, focusing on the quantification of detention time and effective volume. However, only the length-to-width ratio effect was analysed, without accounting for the combined influence of the expansion ratio.

Spreading of a plane turbulent jet into a quiet shallow water body was studied by Giger et al. (1991) for an infinitely-wide

Revision received 15 March 2011/Open for discussion until 31 December 2011. 
expansion. Most published research concerns sudden plane expansions of infinite length (Abbott and Kline 1962), thereby neglecting the effect of the length-to-width ratio on the flow pattern, which strongly influences the onset of flow-field instability. An increase of the length-to-width ratio can lead to stable asymmetric flow at a constant Reynolds number, as demonstrated by Mizushima and Shiotani (2001) and Dewals et al. (2008). Existing literature mainly focuses on pressurized flow, both in laminar (Durst et al. 1974, Cherdron et al. 1978) and turbulent (Aloui and Souhar 2000) conditions. For example, the effect of the Reynolds number on the transition from symmetric to asymmetric flow pattern was studied. A comprehensive review of turbulent sudden plane expansions is provided by Escudier et al. (2002).

This research presents experimental results carried out in a rectangular free surface shallow reservoir. It completes the tests of Kantoush et al. (2006) on the same laboratory facility and allows for a thorough assessment of the types of flow patterns which develop in a shallow reservoir as a function of its length-to-width ratio and expansion ratio, for fixed hydraulic conditions. A comparison with recent results obtained in a similar experimental facility at Liège University (Dufresne et al. 2010a, 2010b) is also presented. While their experiments focused on reattachment length, the present study aims to measure velocity by Ultrasound Velocity Profilers (UVP). The two horizontal velocity components were measured over the entire reservoir, thus allowing a description of the large coherent eddies. The pre-existing experimental database concerning the types of flow patterns in a shallow reservoir was widely extended and enriched by these new measurements.

\section{Experimental set-up}

The experiments were carried out at the Laboratory of Hydraulic Constructions (LCH) of Ecole Polytechnique Fédérale de Lausanne (EPFL). The test facility was a rectangular shallow PVC reservoir with a smooth flat bottom of maximum depth $0.3 \mathrm{~m}$, maximum length $L=6 \mathrm{~m}$ and maximum width $B=$ $4 \mathrm{~m}$. The inlet and outlet free surface channels were $b=$ $0.25 \mathrm{~m}$ wide and $l=1 \mathrm{~m}$ long, i.e. $l / h=5$. The limited inlet length did not allow the development of a fully-turbulent velocity profile. A honeycomb was placed at the inlet to generate a nearly uniform velocity distribution over the entire crosssection. Both channels were placed, respectively, at the centre of the up and downstream reservoir sides. Movable PVC walls were allowed to adjust length $L$ and width $B$, to obtain ratios of $L / B$ between 0.5 and 12 , and expansion ratios $B / b$ from 2 to 16 . The basin was fed with a constant discharge $Q$. The reservoir water depth $h$ was controlled by a flap gate placed at the downstream chamel end.

The water depth was fixed at $h=0.20 \mathrm{~m}$ and the discharge at $Q=71 / \mathrm{s}$, corresponding to an inflow (subscript in) Froude number of $F_{i n}=V /(g h)^{1 / 2}=0.10$ and a Reynolds number of
$\mathrm{R}_{\mathrm{in}}=4 \rho V_{\mathrm{in}} h / \mu=112,000$ (Chanson 1999). The non-dimensional inlet water depth is $h / b=0.80$.

\section{UVP measurements}

Velocity measurements were made to investigate the horizontal velocity field. Since the reservoir depth is much smaller than its horizontal characteristic length scale $(h / L<<1)$, the vertical velocity component can be neglected, as typical for shallow free surface flows (Vreugdenhil 1994); this assumption was also confirmed by additional model measurements.

A horizontal movable square grid of $1 \mathrm{~m} \times 1 \mathrm{~m}$ equipped with 8 UVP transducers $(2 \mathrm{MHz})$ allowed to measure the horizontal velocity components $u$ and $v$ along the transducer axis, four placed along the $x$ direction and four along the $y$ direction (Figure 1). The maximum distance from the instruments was $723 \mathrm{~mm}$ based on the velocity range recordable by the transducer of $\pm 0.189 \mathrm{~m} / \mathrm{s}$ (Metflow 2002). The horizontal velocity vectors were determined at the 16 points formed at the intersections between the velocity profiles recorded by each transducer (Camnasio et al. 2010). The distance between each measurement point was $\sim 24 \mathrm{~cm}$. Each transducer acquired 150 velocity profiles during $80 \mathrm{~s}$; these can be considered to represent the instantaneous velocity. However, the profiles were automatically subdivided by the acquisition software in 15 subsets, resulting in 15 average velocit maps, showing the velocity vector in each of the 16 points of intersection between the velocity profiles of the transducers grid. The final map of the average velocity field was obtained by averaging the 15 velocity maps. After having acquired the data at one reservoir location, the grid was moved to the next position, thus covering step-by-step the entire reservoir surface. For the largest reservoir configuration of $6 \mathrm{~m} \times 4 \mathrm{~m}, 24$ grid positions were necessary, corresponding to 384 measurement points regularly distributed all over the reservoir. Therefore, the recorded velocity field was in principle not instantaneous, but the steady flow condition (constant discharge and depth) and the

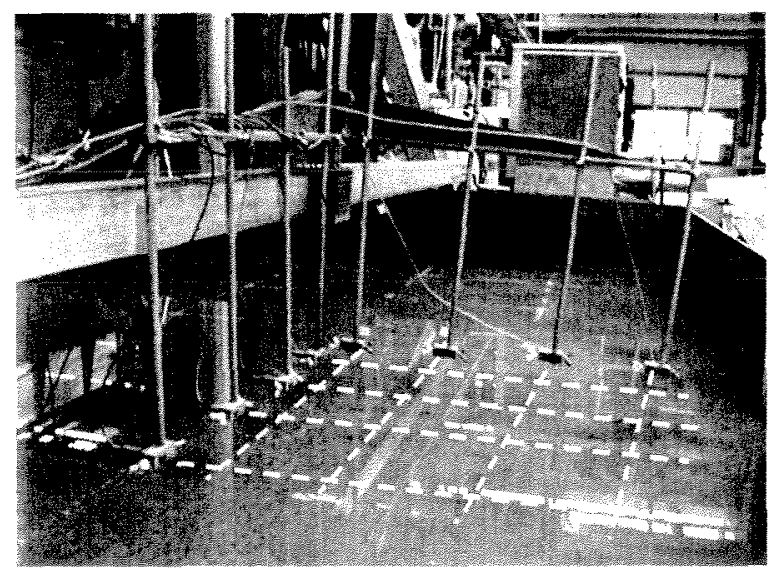

Figure 1 Movable square grid formed by eight UVP transducers utilized for horizontal velocity components measurements. Directions of measured horizontal velocity profles are represented by dashed lines 
macroscopic steadiness of the flow patterns guaranteed the steadiness of the recorded average velocity fields. Longer measurement durations would be necessary in the future to identify more accurately the effective mean velocities, and not only the timeaveraged quantities here presented. By matching the average velocity maps to the reference coordinate system of the reservoir, the average horizontal velocity field was obtained: it allowed to classify the different types of flow patterns as a function of reservoir geometry, indicating also quantitative information on the intensity and the direction of velocity vectors.

A narrow band $0.4 \mathrm{~m}$ wide along the reservoir perimeter could not be experimentally investigated, due to practical problems in grid positioning. However, this portion is negligible compared with the entire reservoir surface. Note that UVP measurements are an invasive method, since transducers are submerged into water, yet in this case the transducer presence did not affect the measured flow fields. Since velocities are small, the transducer influences velocity data only within its vicinity, whose radius is much smaller than the distance of the first measurement point from the transducer. Horizontal velocity vectors at various heights $z$ from the reservoir bottom $(z=0.01,0.06$, $0.11,0.18 \mathrm{~m}$ ) and in several reservoir locations were measured, to define the average shape of the vertical profile versus distance $z$. Since the average velocity profile is logarithmic, it was decided to set the UVP transducers at height $z=0.08 \mathrm{~m}(0.4 h)$, so that the measured velocity represents the mean velocity (Graf and Altinakar 2008).

UVP measurements of the vertical velocity component $w(z)$ were carried out as well, as the flow is in principle not completely two-dimensional (Abbott and Kline 1962, Casarsa and Gianattasio 2008). To apply the shallow-water theory, the order of magnitude of the vertical velocity must be inferior or equal to the order of magnitude of the product between the horizontal velocity scale and $h / L$ (Pedlosky 1979). In the present case, $h / L=\mathrm{O}\left(10^{-2}\right)$, the horizontal velocity scale is $\mathrm{O}\left(10^{-1}\right) \mathrm{m} / \mathrm{s}$, so the vertical velocity component must be of the order of some $\mathrm{mm} / \mathrm{s}$, as confirmed by measurements. Therefore, the horizontal velocity components are sufficient to describe the main characteristics of the reservoir flow field, in particular the large-scale turbulent structures, which have a vertical axis. These turbulent structures have dimensions of the order of the reservoir length scale $L$, they contain the main portion of the kinetic energy and depend on the geometric boundary conditions (Versteeg and Malalasekera 1995).

\section{Experimental results}

The classifications of the flow patterns that develop in the reservoir for the 41 tests are shown in Figure 2 as a function of $L / B$ and $B / b$. The configurations tested by Dufresne et al. (2010a) for the same test conditions, and by Kantoush (2008) in the same LCY facility, are also included. Note that the additional data provided in the present work give a more complete view

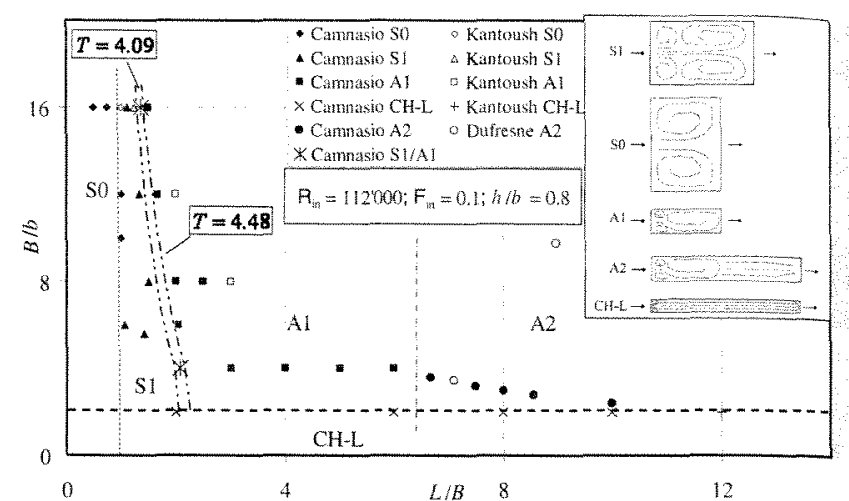

Figure 2 Classification of symmetrical ( $\mathrm{S} 0$ and S1), asymmetrical ( $\mathrm{Al}$ and $\mathrm{A} 2$ ), unstable (Al/S1) and channel-like (CH-L) flow patterns resulting from EPFL tests by Kantoush (2008) and Camnasio (present study), and from Dufresne et al. (2010a) under identical test conditions for $\mathrm{R}_{\mathrm{in}}=112,000, \mathrm{~F}_{\mathrm{in}}=0.10$ and $h / b=0.8$

of the geometrical effect on flow patterns, because Kantoush's tests concerned only eight configurations, with fixed values of $L=6 \mathrm{~m}$ and $B$ varying between 0.5 and $4 \mathrm{~m}$, or $B=4 \mathrm{~m}$ and $L$ varying between 3 and $6 \mathrm{~m}$. In contrast, the present tests cover the entire range of combinations of reservoir widths and lengths feasible at the LCH facility, allowing the analyses of the combined effects of $L / B$ and $B / b$. Figure 2 can be divided into three main zones, corresponding to different flow patterns:

- Channel-like flow (CH-L), characterized by the smallest range of expansion ratios (maximum $B / b=2$, corresponding to $B$ $=0.5 \mathrm{~m}$ ), independent from reservoir length $L$. This flow field has no large recirculation zones, except for two small eddies on both sides of the inflow jet. The flow field across most of the reservoir is one-dimensional, characterized by a constant velocity profile. This type of flow field was observed also by Abbott and Kline (1962), who found that an asymmetrical flow pattern develops if $B / b>2.67$. However, their tests were such that the length $L$ of the expanded reservoir portion could be considered infinite with respect to the expansion width $B$, so that the effect of the length-to-width ratio had not been analysed.

- Symmetric flow (S0 and S1) generated for sufficiently small $L / B$. The critical threshold $L / B$ value below which a stable symmetric flow field develops is a function of $B / b$; the critical $L / B$ value decreases with $B / b$. The threshold is identified in Fig. 2 by the dashed line corresponding to the critical nondimensional shape parameter $T=L /\left[(B-b)^{0.6} \cdot b^{0.4}\right]=4.09$ of Dufresne et al. (2010a). This was confirmed by the present tests (Fig. 2). Within the symmetric flow region, two subsets are identified, namely the two-eddies flow field SO, which develops if $L / B \leq 1$, and the four-eddies flow field $\mathrm{S} 1$, which develops otherwise. The symmetric flow fields $\mathrm{SO}$ and $S I$ are characterized by a main jet traversing straight from the inlet to the outlet along the reservoir axis and by the presence of eddies placed symmetrically with respect to the main jet. For the $S 0$ flow field, only one eddy at both 
sides of the main jet develops, occupying the entire reservoir length, while the S1 flow field is characterized by two eddies on each side of the main jet. In this case, the two upstream eddies, which are smaller and slower than the two downstream eddies, are classified as stagnation zones.

- Asymmetric flow (A1 and A2), characterized by $B / b>2$, and $T>4.48$. Two types $\mathrm{A} 1$ and $\mathrm{A} 2$ of asymmetric flow patterns were observed. For both, the main jet is deflected towards one of the lateral reservoir sides, where it attaches to the wall. Further downstream, the jet flows along the wall to the outlet channel. This particular formation of an asymmetric flow field in a symmetric geometry is attributed to the "Coanda effect" (Wille and Fernholz 1965). The main characteristic of flow field A1 is a large eddy occupying the main reservoir portion, with two smaller eddies in the upstream separated region. In contrast, for flow field $\mathrm{A} 2$, the large eddy does not span over the entire reservoir length and downstream part of the reservoir exhibits a channel-like flow. Dufresne et al. (2010a) also observed the A2 flow pattern under the same hydraulic conditions, as indicated in Fig. 2. Herein, flow field A2 was observed for narrow $(2<B / b<4)$ and long $(L / B$ $>6$ ) reservoirs. However, these tests did not include configurations with $2<B / b<4$ and $L / B<6$. Therefore, additional data are required to identify the conditions necessary for the existence of this flow field. Accordingly, the separation line between the A1 and A2 flow patterns in Fig. 2 must be considered as a preliminary approximation.

Furthermore, two reservoir configurations of "unstable behaviour" were detected. For these configurations, tests indicated a symmetrical flow field S1, while others, carried out under identical test conditions, were characterized by the asymmetric flow pattern A1. These configurations are included in the instability zone of transition between the symmetric flow-field region and the asymmetric flow-field region, which is defined by the two critical values of the non-dimensional shape parameter $T=4.09$ and $T=4.48$, as proposed by Dufresne $e t$ al. (2010a). The flow in this transitional zone is sensitive to external perturbations (Dewals et al. 2008) and results from the phenomenon of bifurcation (Shapira et al. 1990): two solutions of the Navier-Stokes equations exist, one corresponding to the symmetric, the other to the asymmetric flow field. Outside this instability region the flow pattern is stable, in the sense that the type of flow field is not sensitive to external disturbances.

Maps of the average velocity vectors were produced for all the observed types of flow patterns (Figure 3). The time-averaged local horizontal velocity $V=\left(u^{2}+v^{2}\right)^{1 / 2}$, calculated by interpolation of the UVP data, has been normalized by the theoretical reservoir plug-flow velocity $V_{\text {res }}=Q /(B h)$, obtaining the nondimensional (subscript nd) velocity $V_{\text {nd }}=V / V_{\text {res }}$ : at the reservoir inlet, the inflow jet has an average velocity $V_{\text {in }}=0.14 \mathrm{~m} / \mathrm{s}$ equal to that of the inlet channel. For asymmetric flow fields, the velocity of the main jet decreases rapidly as it moves along the reservoir and spreads out (Fig. 3a), reaching values of $\sim 80 \mathrm{~mm} / \mathrm{s}$ in the
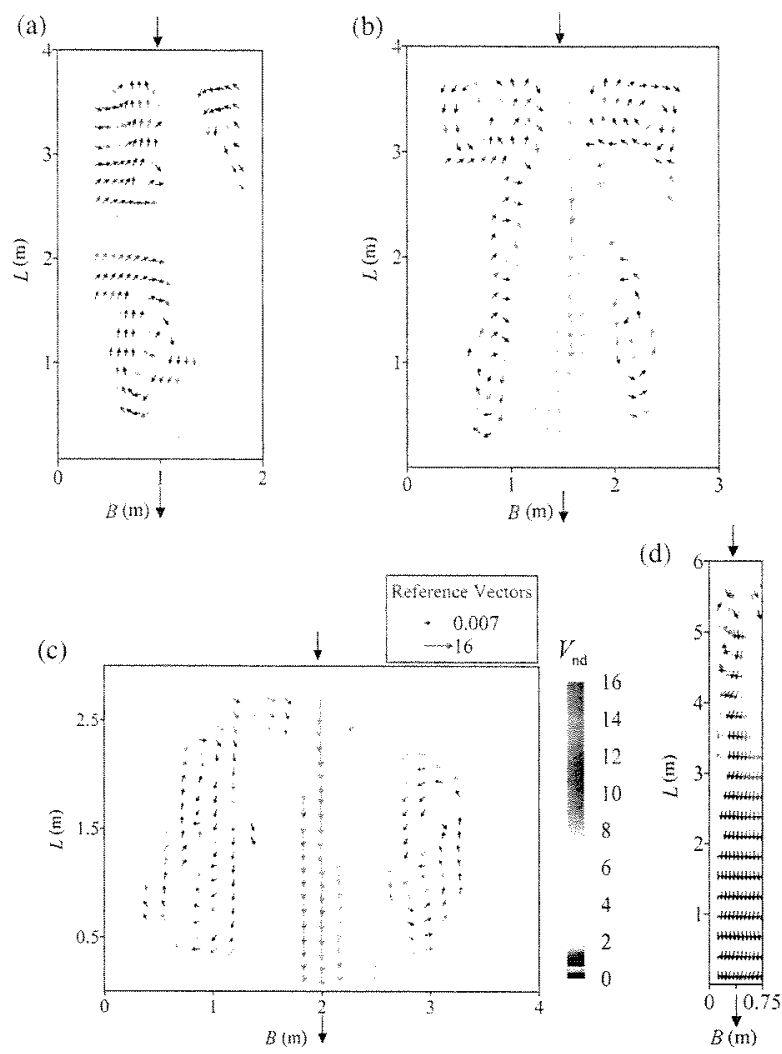

Figure 3 Examples of velocity maps. Normalized velocities $V / V_{\text {res }}$ for various flow patterns types and different reservoir geometry (a) $A 1: L / B$ $=2, B / b=8, V_{\mathrm{res}}=17.5 \mathrm{~mm} / \mathrm{s}$, (b) S1: $L / B=1.33, B / b=12, V_{\mathrm{res}}$ $=11.6 \mathrm{~mm} / \mathrm{s}$, (c) S0: $L / B=0.75, B / b=16, V_{\text {res }}=8.7 \mathrm{~mm} / \mathrm{s}$ (d) A2: $L / B=8, B / b=3, V_{\text {res }}=46 \mathrm{~mm} / \mathrm{s}$ (complete test data are available upon request to authors)

downstream part of the main jet. In contrast, for symmetric flow fields, especially for S0 flow (Fig. 3c), the jet diffusion is smaller, and the velocity remains nearly constant and high at $\sim 120 \mathrm{~mm} / \mathrm{s}$ along the main jet, with high velocity gradients between the main jet and the recirculation zones.

For all cases, the recirculation core is characterized by velocities of the order of $\sim 10 \mathrm{~mm} / \mathrm{s}$. For flow fields A1, S0 and $\mathrm{S} 1$, the velocities are near to the theoretical average velocity $V_{\text {res }}\left(V_{\text {nd }} \approx 1\right)$ in the core of the eddies, while velocities are more than ten times higher than $V_{\text {res }}$ along the main jet. However, an approximately uniform velocity distribution over the entire cross-section (i.e. a plug-flow condition) results for the A2 flow pattern (Fig. 3d), beyond an initial asymmetry of the main jet at the reservoir entrance.

It is concluded that a stable symmetric flow field exists only for sufficiently short reservoirs ( $L / B<1.5$ to 2 , depending on $B / b$, according to the critical threshold of the $T$ shape parameter). For a fixed reservoir width, the flow field changes from asymmetrical Al to symmetrical SI and then to S0 as the reservoir length is reduced. In other words, a reservoir length reduction leads the flow pattern towards symmetry. The flow field symmetry is also infuenced by the proximity of the lateral walls to the main jet: a reservoir length characterized by symmetric flow fields $\$ 1$ or $S 0$ 
at large reservoir width presents a flow field that changes from symmetric to asymmetric Al as the reservoir is narrowed. Moving the lateral walls closer to the main jet has thus a destabilizing effect on the flow field, in agreement with the Coanda effect (Wille and Fernholz 1965, Sobey and Drazin 1986 , Chiang et al. 2000). If the reservoir is narrowed, the symmetric flow pattern becomes first unstable and then definitely asymmetric. A lateral wall proximity, therefore, can prevail over the stabilizing effect due to the closeness of the outlet to the inlet, which would favour the flow pattern symmetry. If the reduction of reservoir width is taken to the extremes, the flow field regains symmetry (A2 flow field and then channel-like flow), but now the reservoir flow tends towards the one-dimensional channel flow, with uniform cross-sectional velocity distribution and without large recirculation zones.

\section{Comparison with recent experimental studies}

In Figure 4, the EPFL results and these of Dufresne et al. (2010a) are compared. Both studies concerned flow-field typology in rectangular shallow reservoirs with symmetrical inlets and outlets, and the authors partly investigated the same geometrical domain. While velocity was measured over the entire reservoir at EPFL, allowing to define streamlines and average velocities, Dufresne et al. focused on the variability of the reattachment length. A classification of possible flow patterns was also given, by suggesting the cited criterion to distinguish between symmetrical and asymmetrical flow patterns. The geometric configurations tested herein cover a wide range of combinations of reservoir length-to-width ratio and expansion ratio, extending the range of geometries tested by Kantoush, while Dufresne et al.'s tests mainly analysed the region of transition between asymmetric and symmetric flow fields for $2<B / b<4$ and 1 $<L / B<3$. Furthermore, the latter also performed a sensitivity analysis of the non-dimensional parameters $h / B, \mathrm{R}_{\mathrm{in}}$ and $\mathrm{F}_{\mathrm{in}}$. Therefore, not all the data shown in Figure 4 correspond to the same values of $h / B, \mathrm{R}_{\mathrm{in}}$ and $\mathrm{F}_{\mathrm{in} \text {. }}$

The geometrical $(h / b$ or $h / B)$ and hydraulic $\left(\mathrm{R}_{\mathrm{in}}\right.$ and $\left.\mathrm{F}_{\mathrm{in}}\right)$ parameters influence the flow-field typology. For example, Dufresne et al. (2010a) found that the flow field changes from

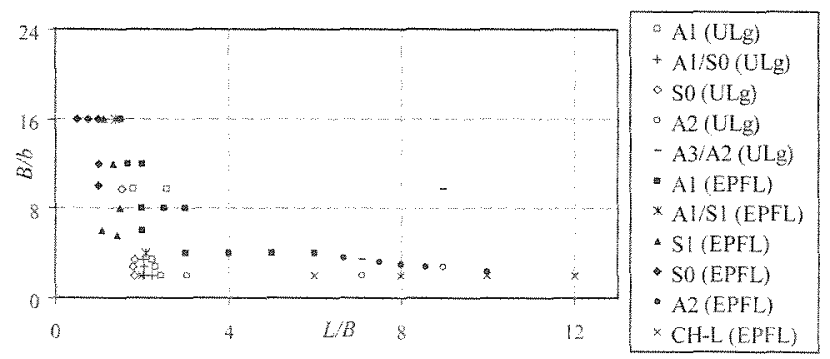

Figure 4 Overview of tests performed at EPFL (present study and Kantoush 2008) and by Dufresne et al. (2010a), resulting in symmetrical (SO and S1), asymmetrical (A1, A2 and $\mathrm{A} 3$ ), and channel-like (CH-L) flow patterns
A2 to $\mathrm{A} 3$ if $h / B<0.1$. Their A3 type is similar to the $\mathrm{A} 2$ flow field but characterized by a further recirculation zone in the downstream reservoir portion. To analyse exclusively the effect of length-to-width and expansion ratios, only tests conducted under identical hydraulic conditions and $h / b$ are plotted in Figure 2. Note from Figure 4 that the transition region from asymmetric to symmetric flows occurs if $1.5<L / B<2.2$, depending on the expansion ratio, though Dufresne et al.'s tests neither had the same hydraulic conditions, nor the same $h / b$ ratio. The critical $T$ values of Dufresne et al. (2010a) for the transitional zone are confirmed by the present tests. Further, these new results also show a channel-like flow independent of the length-to-width ratio at $B / b=2$, in agreement with Abbott and Kline (1962). In contrast, Dufresne et al. (2010a) found for $B / b=2.031$ different flow patterns (S0, A1, A2) depending on the length-to-width ratio. This discrepancy may be due to the different hydraulic conditions at $B / b \sim 2$, namely $F_{\text {in }}=0.2$ by Dufresne et al., while $F_{\text {in }}=0.1$ at EPFL.

\section{Conclusions}

Average velocity fields and streamlines for a wide range of rectangular shallow reservoirs were obtained. In agreement with previous works, different types of flow patterns were identified, including channel-like flow, two symmetric flow patterns characterized by two or four large eddies, and two asymmetrical flow patterns. Their development is due to the combined effect of reservoir expansion and length-to-width ratios. Additional reservoir configurations were tested to confirm and generalize the results. Therefore, a flow pattern corresponding to a certain reservoir configuration can be predicted based on the non-dimensional reservoir length and width. Average velocity fields indicate large gradients between the main jet and the recirculation zones for all flow patterns, except for the reservoir configurations which tend to the one-dimensional channel. The agreement between the present results and those of Dufresne et al. (2010a) confirms the validity of the performed tests strengthening confidence in the results, as they emerge from two independent facilities.

\section{Acknowledgements}

S. Kantoush provided helpful support and suggestions on experimentation. Thanks to M. Dufresne and B. Dewals for fruitful discussions on this research study.

\section{Notation}

$B \quad=$ reservoir width $(\mathrm{m})$

$b=$ width of inlet channel $(\mathrm{m})$

$F=$ Froude number $(-)$

$g=$ gravitational acceleration $\left(\mathrm{m} / \mathrm{s}^{2}\right)$ 
$h=$ water depth $(\mathrm{m})$

$L=$ reservoir length $(\mathrm{m})$

$l=$ length of inlet and outlet channels $(\mathrm{m})$

$R=$ Reynolds number $(-)$

$T=$ non-dimensional reservoir shape parameter $(-)$

$u=$ horizontal velocity component in $x$ direction $(\mathrm{m} / \mathrm{s})$

$v=$ horizontal velocity component in $y$ direction $(\mathrm{m} / \mathrm{s})$

$V=$ horizontal velocity $(\mathrm{m} / \mathrm{s})$

$V_{\mathrm{res}}=$ theoretical mean plug flow velocity in the reservoir $(\mathrm{m} / \mathrm{s})$

$w=$ vertical velocity component $(\mathrm{m} / \mathrm{s})$

$x=$ stream-wise coordinate $(\mathrm{m})$

$y=$ transverse coordinate $(\mathrm{m})$

$z=$ vertical coordinate $(\mathrm{m})$

$\mu=$ water viscosity $(\mathrm{kg} /(\mathrm{m} \cdot \mathrm{s}))$

$\rho=$ water density $\left(\mathrm{kg} / \mathrm{m}^{3}\right)$

\section{Subscripts}

in inlet

nd non-dimensional

\section{References}

Abbott, D.E., Kline, S.J. (1962). Experimental investigation of subsonic turbulent flow over single and double backward facing steps. J. Basic Engng. 84(9), 317-325.

Aloui, F., Souhar, M. (2000). Experimental study of turbulent asymmetric flow in a flat duct symmetric sudden expansion. J. Fluid. Eng. 122(1), 174-177.

Camnasio, E., De Cesare, G., Kantoush, S. (2010). Flow field and sediment deposition in a rectangular shallow reservoir with non symmetric inlet and outlet configuration. 7th ISUD Conf. Gothenburg, Sweden, 53-56.

Casarsa, L., Giannattasio, P. (2008). Three-dimensional features of the turbulent flow through a planar sudden expansion. Physics of Fluids 20(1), 1-15.

Chanson, H. (1999). The hydraulics of open channel flow. Butterworth-Heinemann, Oxford UK.

Cherdron, W., Durst, F., Whitelaw, J.H. (1978). Asymmetric flows and instabilities in symmetric ducts with sudden expansions. J. Fluid Mech. 84(1), 13-31.

Chiang, T.P., Sheu, T.W.H, Wang, S.K. (2000). Side wall effects on the structure of laminar flow over a plane-symmetric Sudden expansion. Computers and Fluids 29(6), 467-492.

Dewals, B.J., Kantoush, S.A., Erpicum, S., Pirotton, M., Schleiss, A.J. (2008). Experimental and numerical analysis of flow instabilities in rectangular shallow basins. Env. Fluid Wech. $8(1), 31-54$.

Dufesne, M., Dewals, B.J., Erpicum, S., Archambeau, P., Piroton, M. (2010a). Classification of how patterns in rectangular shallow reservoirs. J. Hydraulic Res. 48(2), $197-204$.
Dufresne, M., Dewals, B.J., Erpicum, S., Archambeau, P., Pirotton, M. (2010b). Experimental investigation of flow pattern and sediment deposition in rectangular shallow reservoirs. Int. J. Sediment Res. 25(3), 258-270.

Durst, F., Melling, A., Whitelaw, J.H. (1974). Low Reynolds number over a symmetrical sudden expansion. J. Fluid Mech. 64, 111-128.

Escudier, M.P., Oliveira, P.J., Poole, R.J. (2002). Turbulent flow through a plane sudden expansion of modest aspect ratio. Phys. Fluids 14(10), 3641-3654.

Frey, P., Champagne, J.Y., Morel, R., Gay, B. (1993). Hydrodynamics fields and solid particles transport in a settling tank. J. Hydraulic Res. 31(6), 736-776.

Giger, M., Dracos, T., Jirka, G.H. (1991). Entrainment and mixing in plane turbulent jets in shallow waters. $J$. Hydraulic Res. 29(5), 615-642.

Graf, W.H., Altinakar, M. (2008). Hydraulique fuviale: Ecoulement et phénomènes de transport dans les canaux à géométrie simple. Presses Polytechniques et Universitaires Romandes, Lausanne.

Kantoush, S.A., Bollaert, E., De Cesare, G., Boillat, J.-L., Schleiss, A.J. (2006). Flow field investigation in a rectangular shallow reservoir using UVP, LSPIV and numerical model. Proc. 5th Int. Symp. Ultrasonic Doppler Meth. for Fluid Mech. and Fluid Eng. ETH Zurich, Switzerland, 129-134.

Kantoush, S.A. (2008). Experimental study on the influence of the geometry of shallow reservoirs on flow patterns and sedimentation by suspended sediments. PhD Thesis 4048 . EPFL, Lausanne.

Kantoush, S.A., Schleiss, A.J. (2009). Channel formation during flushing of large shallow reservoirs with different geometries. Env. Techn. 30(8), 855-863.

Metflow, SA (2002). UVP Monitor: Model UVP-DUO with software version 3. User's Guide. Lausanne, Switzerland.

Mizushima, J., Shiotani, Y. (2001). Transitions and instabilities of flow in a symmetric channel with a suddenly expanded and contracted part. J. Fluid Mech. 434, 355-369.

Oca, J., Masaló, I., Reig, L. (2004). Comparative analysis of flow patterns in aquaculture rectangular tanks with different water inlet characteristics. Aquacultural Eng. 31(3-4), 221-236.

Pedlosky, J. (1979). Geophysical Fluid Dynamics, 57-63. Springer, New York.

Persson, J. (2000). The hydraulic performance of ponds of various layouts. Urban Water 2(3), 243-250.

Persson, J., Wittgren, H.B. (2003). How hydrological and hydraulic conditions affect performance of ponds. Ecol. Eng. 21(12), 259-269.

Shapira, M., Degani, D., Weihs, D. (1990). Stability and existence of multiple solutions for viscous flows in suddenly enlarged channels. Comp. and Fluids 18(3), 239-258.

Sobey, I.J. Drazin, P.G. (1986). Bifurcation of 2 D channel fows. J. Fluid Mech. 171, 263-287.

Stovin, VR, Saul, A.J. (1994). Sedimentation in storage tank structures. Water Sci. and Tech. 29(1-2), 363-372. 
Thackston, E.L, Shields, F.D., Schoeder, P.R. (1987). Residence time distributions of shallow basins. J. Environ. Eng. 113(6), 1319-1332.

Versteeg, H.K., Malalasekera, W. (1995). An introduction to computational fluid dynamics 3: The finite volume method. Longman, Essex.
Vreugdenhil, C.B. (1994). Numerical methods for shallow-water flow, 21-25. Kluwer Academic, Dordrecht.

Wille, R., Fernholz, H. (1965). Report on the first European Mechanics Colloquium, on the Coanda effect. $J$. Fluid Mech. 23(4), 801-819. 